\title{
The Nature of Naked Singularity in Cosmology
}

\section{Haradhan Kumar Mohajan*}

Premier University, Chittagong, BANGLADESH

*Corresponding Contact:

Email: haradhan1971@gmail.com

\begin{abstract}
This paper discusses aspects of a naked singularity. If a star has exhausted its nuclear fuel, and it exceeds Chandrasekhar limit then it must undergo gravitational collapse and ultimately it forms black hole. A black hole is a space from where no massive particle or no signal comes to the external observer. As a result space-time singularity is formed; this must be hidden behind the black hole region which is called the cosmic censorship hypothesis, first proposed by Roger Penrose, which cloths the singularity to remain invisible to the external observers. It has not been possible, to obtain a proof despite many attempts to establish the validity of cosmic censorship and it remains an open problem. Hence, general relativity supports that naked singularity may be formed. A naked singularity implies that singularity is visible to an external observer. Sometimes it is not visible to the external observers, but it could be observable to the interior observers of the black hole region. This type of singularity is called local naked singularity.
\end{abstract}

Key words

Cosmic censor, Einstein equation, gravitational focusing, naked singularity, Vaidya region

\section{INTRODUCTION}

In 1969, Roger Penrose first posed a hypothesis that there exists a cosmic censor hypothesis that forbids occurrence of naked singularities. There are two versions of cosmic censorship: i) a strong one and ii) a weak one (Penrose 1969). The strong version says that space-time is globally hyperbolic. The weak version says that the intersection of the causal future of a partial Cauchy surface and the causal past of the boundary at infinity is globally hyperbolic (Kr'olak 1999).

Space-time singularities can be divided into two kinds, according to whether or not they can be observed. An observable space-time singularity is called a naked singularity that is not hidden by horizons, and a space-time singularity that cannot be observed is a black hole singularity. The horizon is the boundary of the black hole region which is cutoff from the outside world. The appearance of a naked singularity represents the formation of an observable high-curvature, strong-gravity region (Harada et al. 2002). 
Singularities also form without or before formation of a trapped surface or an event horizon. If such a singularity forms, then non-spacelike geodesics come out of it and in principle the singularity can be visible to an outside observer. There is a conjecture that gamma ray bursts originate from such naked singularities (Witten 1992).

The singularity theorems do not prove that collapse will definitely end in a black hole. The alternative to a black hole is a naked singularity. If a singularity is naked, light rays starting at the singularity could escape all the way to infinity. Both black holes and naked singularities can form in collapse, depending on the choice of initial conditions (Singh 1997). According to Lorentzian geometry naked singularity is a timelike ideal point of the boundary of space-time that is visible for the existence of the outgoing radial null geodesics from the singularity (Hawking and Ellis 1973).

At present there are many investigations supporting the cosmic censor hypothesis as well as many examples of existence of naked singularities. We cannot predict laws of physics at a singularity; hence existence of a naked singularity can lead to breakdown of predictability. Hence naked singularities would be a disaster for general relativity and as well as for physics (Christodoulou 1999, Harada 2004, Deshingkar 2010).

\section{ObJectives OF The Study}

The objectives of the study are to discuss naked singularity and also black hole singularity. At present it is not known that the singularity must be either hidden within an event horizon of gravity or visible to the external observers. To discuss the naked singularity we have first highlighted on general relativity and discuss briefly the gravitational collapse of a massive star. If a star has exhausted its nuclear fuel then it must undergo gravitational collapse. Roger Penrose's cosmic censor hypothesis forbids the occurrence of naked singularities. But cosmic censor hypothesis has not been established in physics with strong evidence. So, the demand of naked singularity is not illogical in general relativity. The objective of the study is to make easier to the common readers to understand the concept of naked singularity.

\section{Highlights on General ReLativity}

By the covariant differentiations of vectors, we can write the relation (Mohajan 2014a);

$$
A_{\mu ; \nu ; \sigma}-A_{\mu ; \sigma ; v}=R_{\mu \nu \sigma}^{\alpha} A_{\alpha},
$$

where $R_{\mu v \sigma}^{\alpha}=\Gamma_{\mu \sigma ; v}^{\alpha}-\Gamma_{\mu v ; \sigma}^{\alpha}+\Gamma_{\beta v}^{\alpha} \Gamma_{\mu \sigma}^{\beta}-\Gamma_{\beta \sigma}^{\alpha} \Gamma_{\mu v}^{\beta}$,

is a tensor of rank four and called Riemann curvature tensor. From (1) we observe that the curvature tensor components are expressed in terms of the metric tensor and its second derivatives. From (2) we get;

$$
R_{[\mu \nu \sigma]}^{\alpha}=0 .
$$

Taking inner product of both sides of (2) with $g_{\rho \alpha}$ we get covariant curvature tensor;

$$
R_{\rho \mu v \sigma}=\frac{1}{2}\left(\frac{\partial^{2} g_{\rho \sigma}}{\partial x^{v} \partial x^{\mu}}+\frac{\partial^{2} g_{\mu \nu}}{\partial x^{\sigma} \partial x^{\rho}}-\frac{\partial^{2} g_{\mu \sigma}}{\partial x^{v} \partial x^{\rho}}-\frac{\partial^{2} g_{\rho \nu}}{\partial x^{\sigma} \partial x^{\mu}}\right)+g_{\alpha \lambda}\left(\Gamma_{\mu \nu}^{\alpha} \Gamma_{\rho \sigma}^{\lambda}-\Gamma_{\mu \omega}^{\alpha} \Gamma_{\rho \nu}^{\lambda}\right)
$$

Contraction of curvature tensor (2) gives Ricci tensor; 


$$
R_{\mu v}=g^{\lambda \sigma} R_{\lambda \mu \sigma v} .
$$

Further contraction of (5) gives Ricci scalar;

$$
\hat{R}=g^{\lambda \sigma} R_{\lambda \sigma} .
$$

From which one gets Einstein tensor as;

$$
G_{v}^{\mu}=R_{v}^{\mu}-\frac{1}{2} \delta_{v}^{\mu} R
$$

where $\operatorname{div}\left(G_{v}^{\mu}\right)=G_{v ; \mu}^{\mu}=0$. The space-time $(M, g)$ is said to have a flat connection if and only if;

$$
R_{v \lambda \sigma}^{\mu}=0 \text {. }
$$

This is necessary and sufficient condition for a vector at a point $p$ to remain unaltered after parallel transported along an arbitrary closed curve through $p$. This is because all such curves can be shrunk to zero, in which case the space-time is simply connected (Hawking and Ellis 1973).

The energy-momentum tensor $T^{\mu v}$ is defined as;

$T^{\mu v}=\rho_{0} u^{\mu} u^{v}$

where $\rho_{0}$ is the proper density of matter, and if there is no pressure. A perfect fluid is characterized by pressure $p=p\left(x^{\mu}\right)$, then we get;

$T^{\mu v}=(\rho+p) u^{\mu} u^{v}+p g^{\mu v}$.

The principle of local conservation of energy and momentum states that;

$T_{; v}^{\mu v}=0$.

According to the Newton's law of gravitation, the field equations in the presence of matter are;

$$
\nabla^{2} \phi=4 \pi G \rho
$$

where $\phi$ is the gravitational potential, $\rho$ is the scalar density of matter, $G$ is the gravitational constant. By (12) the Einstein's field equation can be written as;

$$
R_{\mu v}-\frac{1}{2} g_{\mu v} R=-\frac{8 \pi G}{c^{4}} T^{\mu v} .
$$

where $G=6.673 \times 10^{-11} \mathrm{~m}^{3} \mathrm{~kg}^{-1} \mathrm{~s}^{-2}$ is the gravitational constant and $c=10^{8} \mathrm{~m} / \mathrm{s}$ is the velocity of light. Einstein introduced a cosmological constant $\Lambda(\approx 0)$ for static universe solutions as; 


$$
R_{\mu v}-\frac{1}{2} g_{\mu v} R+\Lambda g_{\mu v}=-\frac{8 \pi G}{c^{4}} T^{\mu v}
$$

In relativistic unit $G=c=1$, hence in relativistic units (14) becomes;

$$
R_{\mu \nu}-\frac{1}{2} g_{\mu \nu} R=-8 \pi T_{\mu \nu}
$$

It is clear that divergence of both sides of (14) and (15) is zero. For empty space $T_{\mu v}=0$, so that, $R_{\mu v}=\Lambda g_{\mu v}$, then;

$$
R_{\mu \nu}=0 \text { for } \Lambda=0
$$

which is Einstein's law of gravitation for empty space.

\section{Definitions of Singularity and NaKed Singularity}

First we discuss some definitions related with singularity as follows (Mohajan 2013a):

Definition: The generalized affine parameter (g.a.p.) length of a curve $\gamma:[0, a) \rightarrow M$ with respect to a frame,

$\mathbf{E}=(\underset{a}{\mathbf{E}}, a=0,1,2,3)$

at $\gamma(0)$ is given by;

$$
\ell_{\mathbf{E}}(\gamma)=\int_{0}^{a}\left(\sum_{i=0}^{3} g\left(\dot{\gamma} E_{i}(s)\right)\right)^{1 / 2} d s
$$

where $\dot{\gamma}=\frac{d \gamma}{d s}$ is tangent vector and $\mathbf{E}(s)$ is defined by parallel propogation along the curve, starting with an initial value $\mathbf{E}(0)$.

Definition: A curve $\gamma:[0, a) \rightarrow M$ is incomplete if it has finite g.a.p. length with respect to some frame $\mathbf{E}$ at $\gamma(0)$. If $\ell_{E}(\gamma)<\infty$, then if we take any other frame $\mathbf{E}^{\prime}$ at $\gamma(0)$ we have $\ell_{E^{\prime}}(\gamma)<\infty$. This is because the corresponding parallel propogated frames satisfy;

$E_{i}^{\prime}=L_{i}^{j} E_{j}$

for a constant Lorentz matrix $\mathbf{L}$ and hence;

$$
\ell_{E^{\prime}} \leq\|\mathbf{L}\| \ell_{E^{\prime}}
$$

where $\|\mathbf{L}\|=\operatorname{Sup}\left(\left(L_{i}^{j} X^{i}\right)\right)^{1 / 2}$. 
Definition: A curve $\gamma:[0, a) \rightarrow M$ is termed inextensible if there is no curve $\gamma^{\prime}:[0, b) \rightarrow M$ with $b>a$ such that $\gamma^{\prime}[[0, a)=\gamma$. This is equivalent to saying that there is no point $p$ in $M$ such that $\gamma(s) \rightarrow p$ as $s \rightarrow a$ i.e., $\gamma$ has no end point in $M$.

Einstein's empty space equation (16) is $R_{\mu v}=0$. From this we can the Schwarzschild metric;

$d s^{2}=-\left(1-\frac{2 m}{r}\right) d t^{2}+\left(1-\frac{2 m}{r}\right)^{-1} d r^{2}+r^{2}\left(d \theta^{2}+\sin ^{2} \theta d \phi^{2}\right)$

where there are two singularities at $r=0$ and $r=2 m$, because one of the $g^{\mu v}$ or $g_{\mu v}$ is not continuously defined. Here $r=0$ is a real singularity in the sense that along any non-spacelike trajectory falling into the singularity as $r \rightarrow \infty$, the Kretschman scalar $\alpha=R^{\mu v \gamma \sigma} R_{\mu v \gamma \sigma}$ tends to infinity and $r=2 m$ is a coordinate singularity and could be removed by the coordinate transformation. After some efforts it is realized that $r=2 m$ is not a genuine space-time singularity but merely a coordinate defect, and what was really needed was an extension of the Schwarzschild manifold. Such an extension of the spacetime was obtained by Kruskal and Szekeres (Kruskal 1960, Szekeres 1960) and this may be regarded as an important insight involving a global approach.

Let us consider the metric;

$d s^{2}=-\frac{1}{t^{2}} d t^{2}+d x^{2}+d y^{2}+d z^{2}$

which is singular on the plane $t=0$. If any observer starting in the region $t>0$ tries to reach the surface $t=0$ by traveling along timelike geodesics, he will not reach at $t=0$ in any finite time, since the surface is infinitely far into the future. If we put $t^{\prime}=\log (-t)$ in $t<0$ then (18) becomes;

$d s^{2}=-d t^{\prime 2}+d x^{2}+d y^{2}+d z^{2}$

with $-\infty<t^{\prime}<\infty$ which is Minkowski metric and there is no singularity at all, which is a removable singularity like Schwarzschild singularity at $r=2 m$ (Mohajan 2013c). Let us consider a non-spacelike geodesic which reaches the singularity in a proper finite time. Such a geodesic will have not any end point in the regular part of the space-time. A timelike geodesic which, when maximally extended, has no end point in the regular spacetime and which has finite proper length, is called timelike geodesically incomplete (Joshi 2013).

The Friedmann-Robertson-Walker (FRW) model is given by (Mohajan 2013b);

$$
d s^{2}=-d t^{2}+S^{2}(t)\left[\frac{d r^{2}}{1-k r^{2}}++r^{2}\left(d \theta^{2}+\sin ^{2} \theta d \phi^{2}\right)\right]
$$


where $S(t)$ is the scale factor and $k$ is a constant which denotes the spatial curvature of the three-space and could be normalized to the values $+1,0,-1$.

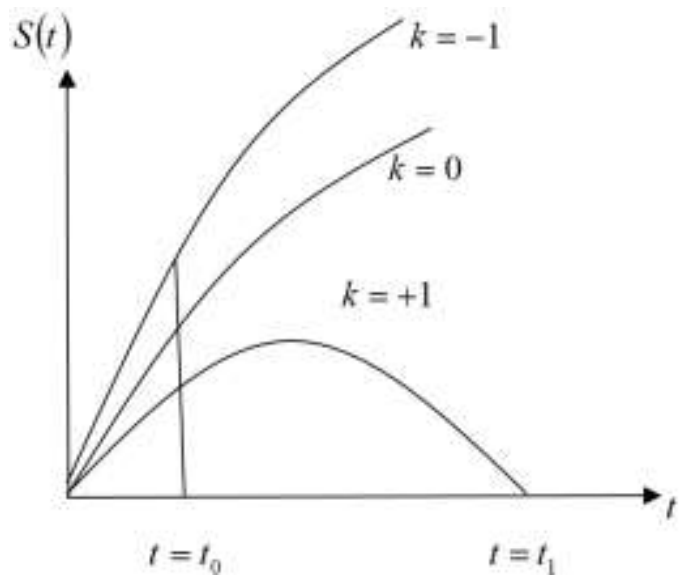

Figure 1: The behavior of the curve $S(t)$ for the three values $k=-1,0,+1$; the time $t=t_{0}$ is the present time and $t=t_{1}$ is the time when $S(t)$ reaches zero again for $k=+1$.

When $k=0$ the three-space is flat and (20) is called Einstein de-Sitter static model, when $k=+1$ and $k=-1$ the three-space are of positive and negative constant curvature; these incorporate the closed and open Friedmann models respectively (figure 1).

The Einstein equations imply that $\rho+3 p>0$ at all times, where $\rho$ is the total density and $p$ is the pressure, there is a singularity at $t=0$, since $S^{2}(t) \rightarrow 0$ when $t \rightarrow 0$ in the sense that curvature scalar $\hat{R}=R^{\mu v} R_{\mu v}$ bends to infinity. Here we consider the time $t=0$ is the beginning of the universe. Thus there is an essential curvature singularity at $t=0$ which cannot be transformed away by any coordinate transformation.

Definition: A space-time is incomplete if it contains an incomplete inextensible curve. By the above definitions we can say that a space-time is called incomplete if it contains an incomplete timelike inextensible curve. The Friedman 'big bang' models are geodesically incomplete, since the curve defined by (Clarke 1993);

$\gamma(s)^{0}=S(t)-s$

$\gamma(s)^{i}=$ Constant $, i=1,2,3$;

is a geodesic which is incomplete, having no endpoint in $M$ as $s \rightarrow S(t)$. Minkowski space is not incomplete. The region $r>2 m$ in the Schwarzschild metric is incomplete, while region $0<r<2 m$ is not a space-time, since the metric is not defined at $r=2 m$.

Definition: An extension of a space-time $(M, g)$ is an isometric embedding $\theta: M \rightarrow M^{\prime}$ where $\left(M^{\prime}, g^{\prime}\right)$ is a space-time and $\theta$ is onto a proper subset of $M^{\prime}$. By 
the above definition Schwarzschild metric is not singular at $r=2 m$ by Kruskal-Szekeres extension (Kruskal 1960, Szekeres 1960, Mohajan 2014a). A space-time is termed extensible if it has an extension.

Definition: A space-time is singular if it contains an incomplete curve $\gamma:[0, a) \rightarrow M$ such that there is no extension $\theta: M \rightarrow M^{\prime}$ for which $\theta \circ \gamma$ is extensible.

A singularity which can causally influence parts of space-time is called a naked singularity. The weak censorship or the strong asymptotic predictability requirement states that the region of space-time outside a black hole must be globally hyperbolic. The weak censorship states that the singularity cannot be globally naked. But it could be locally naked in the sense that an observer within the event horizon and in the interior of black hole could possibly receive particles or photons from the singularity.

\section{Gravitational Collapse}

In 1939, Oppenheimer and Snyder gave the first model of gravitational collapse. They showed that the collapse of a homogeneous dust sphere results in the formation of a black hole. The density of the sphere remains uniform throughout the evolution, and after a certain comoving time the boundary of the star shrinks below the Schwarzschild radius. At a later epoch, all the dust shells shrink to zero radius at exactly the same comoving time. As a result the density diverges and a curvature singularity forms which is hidden behind the event horizon (Singh 1997).

When the star is heavier than a few solar masses, it could undergo an endless gravitational collapse without achieving any equilibrium state. This happens when the star has exhausted its internal nuclear fuel which provides the outwards pressure against the inwards pulling gravitational forces. This creates a black hole in the space-time which covers the space-time singularity. In the Schwarzschild metric, at $r=0$, the curvature and density is infinite, the singularity is completely hidden within the trapped surface region. So, no signal from the singularity could go out to any observer at infinity, and the singularity is disconnected from the outside observers (Joshi 1996, Mohajan 2014b).

\section{Analytical Discussion of Naked Singularity}

In the very final stages of the collapse of the collapsing matter the collapse would be largely radiation dominated. Here we will examine the final fate of such a collapse with special reference to the occurrence of naked singularities and the cosmic censorship hypothesis. For this the following conditions are necessary (Joshi 1996):

The naked singularity has to be visible at least for a finite period of time to any far away observer. The singularity must be gravitationally weak, creating a mere space-time pathology, but must be a strong curvature singularity in a suitable sense (Mohajan 2013a). So, the space-time does not admit any continuous extension through the singularity in a meaningful manner. The form of matter should be reasonable in that it must satisfy a suitable energy condition ensuring the positivity of energy and collapses gravitationally from an initial spacelike hypersurface with a well-defined non-singular initial data. The imploding radiation is described by the Vaidya space-time, given in $(t, r, \theta, \phi)$ coordinates (Vaidya 1943, 1951) as; 
$d s^{2}=-\left(1-\frac{2 m(v)}{r}\right) d v^{2}+2 d v d r+r^{2} d \Omega^{2}$

where $d \Omega^{2}=d \theta^{2}+\sin ^{2} \theta d \phi^{2}$.

The null coordinate $v$ denotes the advanced time and $m(v)$ is an arbitrary non-negative increasing mass function. Equation (21) is also known as the radiating Schwarzschild space-time, describes the geometry outside a radiating spherically symmetric star (Mkenyeleye et al. 2014).

The stress energy tensor for the radial flux of radiation is;

$T_{\mu \nu}=\rho k_{\mu} k_{v}=\frac{1}{4 \pi r^{2}} \cdot \frac{d m}{d v} k_{\mu} k_{v}$,

with $k_{\mu}=-\delta_{\mu}^{v}, k_{\mu} k^{v}=0$,

which represents the radially injected radiation flow into an flat and empty region, which is focused into a central singularity of growing mass by a distance source (figure 2). The source is turned off at a finite time $T$, where the field settles to the Schwarzschild spacetime for $v>T$ with mass $m_{0}=m(T)$ by way of the Vaidya metric (21). Assume $m(v)$ to be a linear function, the central singularity $v=0, r=0$ was studied by Papapetrou (1985) and Kurada (1984), who showed that it will be naked and persistent when the collapse is sufficiently slow. Hiscock et al. (1982) considered the situation when the spacetime admits marginally naked singularity, where the Cauchy horizon coincides with the event horizon allowing only an isolated null trajectory to escape to infinity; and studied the particle creation by such a naked singularity. We now examine the structure and curvature strength of naked singularity arising in the radiation collapse. We specify here all the families of future directed non-spacelike geodesics which might possibly terminate at the singularity $v=0, r=0$ i.e., producing a naked singularity of the space-time. Throughout the present section, we choose $m(v)$ to be a linear function, $2 m(v)=\lambda v$ with $\lambda>0$ (Dwevedi and Joshi 1991). This is the Vaidya-Papapetrou space-time describing radiation collapse. Now we have three regions;

$m(v)=0$ for $v<0$,

$m(v)=\lambda v$ for $0<v<T$, and

$m(v)=m_{0}$ for $v>T$.

Hence the mass for the final Schwarzschild black hole is $m_{0}$ and the causal structure of the space-time depends on the values chosen for the constants $m_{0}, T$ and $\lambda$.

Let, $K^{\mu}=\frac{d x^{\mu}}{d k}$ be tangent to non-spacelike geodesics, where $k$ is the affine parameter, then, 


$$
K_{; \nu}^{\mu} K^{v}=0 \text { and } g_{\mu \nu} K^{\mu} K^{v}=B
$$

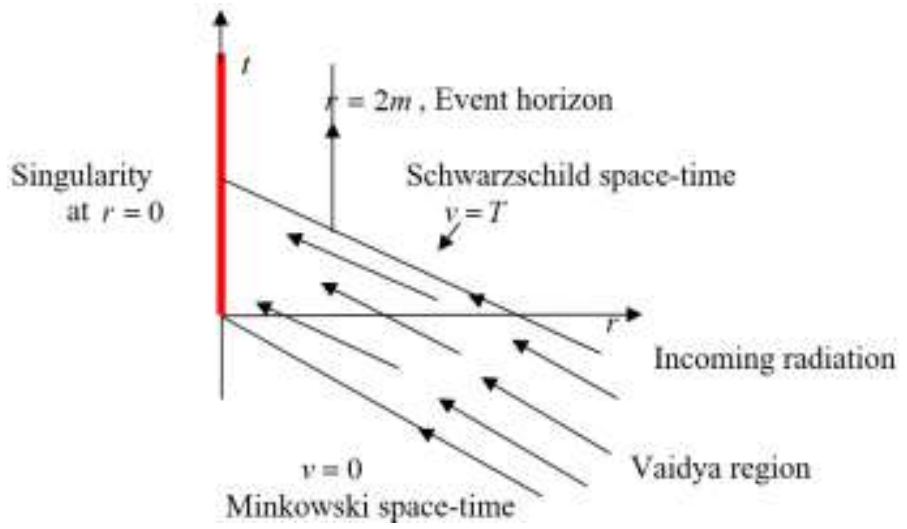

Figure 2: Collapse of a radiation shell at the centre of symmetry. First wave front arrives at $r=0$ at $v=0$ and the final wave front reaches the centre at $v=T$. A singularity is visible at $r=0$. The flat Minkowski space-time for $v<0$ is joined to the Schwarzschild geometry for $v>T$, through the Vaidya region.

where $B=0$ for null vectors and $B= \pm 1$ for spacelike and timelike vectors respectively. Now integrating Lagrange's equation we obtain the components $K^{\theta}$ and $K^{\phi}$ of the tangent vector as,

$$
\begin{aligned}
K^{\theta} & =\frac{\ell \cos \beta}{r^{2} \sin ^{2} \theta}, \\
K^{\phi} & =\frac{\ell \sin \beta \cos \phi}{r^{2}},
\end{aligned}
$$

where $\ell$ is the impact parameter and $\beta$ is the isotropy parameter given by; $\sin \phi \tan \beta=\cot \theta$.

Let us write $2 m(v)=\lambda v$ and defining a new parameter $X=\frac{v}{r}$ gives;

$$
(1-\lambda X) k_{r}^{2}+2 k_{r} k_{v}+\frac{\ell}{r^{2}}=B .
$$

Now we write the general solution as;

$$
\begin{aligned}
& K^{v}=\frac{d v}{d r}=\frac{P(v, r)}{r}, \\
& K^{r}=\frac{d r}{d k}=\frac{(1-\lambda X) P}{2 r}-\frac{\ell^{2}}{2 r P}+\frac{B r}{2 P} .
\end{aligned}
$$

Along radial curve we have $\ell=0$. The function $P$ satisfies the differential equation; 


$$
\frac{d P}{d k}=\frac{P^{2}}{2 r^{2}}(1-2 \lambda X)+\frac{\ell^{2}}{2 r^{2}}+\frac{B}{2}
$$

Dwevedi and Joshi (1989) solved (28) as;

$$
P=\frac{(C+B k) \pm \sqrt{(C+B k)^{2}+\left(\ell^{2}-B r^{2}\right) X\left(2+\lambda X^{2}-X\right)}}{2+\lambda X^{2}-X}
$$

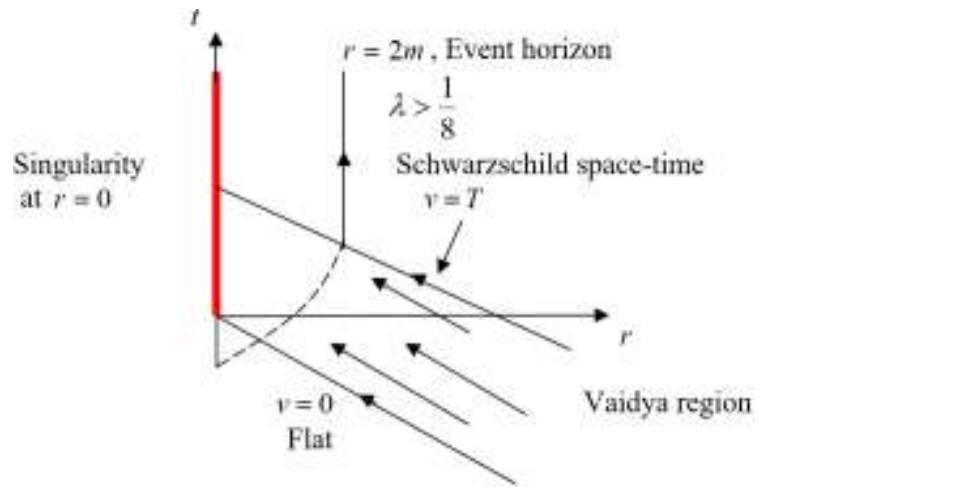

Figure 3: The event horizon completely covers the singularity at $r=0$ when $\lambda>\frac{1}{8}$.

where $C$ is constant and the affine parameter $k=0$ at $r=0, v=0$. From equation (26), (27) and (29) we get;

$$
\frac{d r}{d v}=\frac{(1+A k) \mp\left(1+\lambda X^{2}-X\right) Q}{X((1+A k) \pm Q)}
$$

where we have used $A=B / C, L=\ell / C, r=r(X)$ and

$$
Q=Q(X)=\left[(1+A k)^{2}+\left(L^{2}-\frac{A}{C r^{2}}\right) X\left(2+\lambda X^{2}-X\right)\right]^{1 / 2} .
$$

The point $r=0, v=0$ is a singular point of the above differential equation and is seen to be a naked singularity of the space-time (figure 3 ).

Now, on a singular geodesic let,

$$
\operatorname{Lim}_{v \rightarrow 0, r \rightarrow 0} X=\operatorname{Lim}_{v \rightarrow 0, r \rightarrow 0} \frac{v}{r}=X_{0},
$$

By equation (30) using L' Hospital rule we get;

$$
\begin{aligned}
& X_{0}=\operatorname{Lim}_{v \rightarrow 0, r \rightarrow 0} \frac{v}{r}=\operatorname{Lim}_{v \rightarrow 0, r \rightarrow 0} \frac{(d v / d \lambda)}{(d r / d \lambda)} \\
& =\frac{X_{0}\left(1 \pm Q\left(X_{0}\right)\right)}{\left(1 \mp\left(1+\lambda X_{0}^{2}-X_{0}\right)\right) Q\left(X_{0}\right)}
\end{aligned}
$$


$\Rightarrow\left(2+\lambda X_{0}^{2}-X_{0}\right) Q\left(X_{0}\right)=0$.

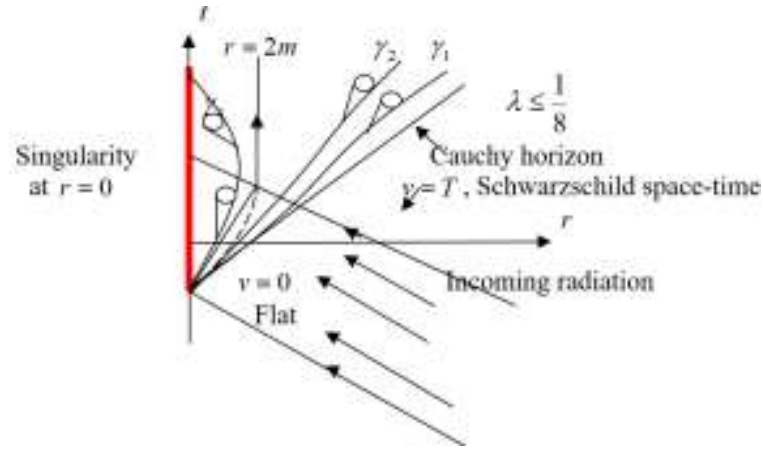

Figure 4: For $\lambda \leq \frac{1}{8}$, a naked singularity forms at the origin. Families of trajectories such as $\gamma_{1}$ and $\gamma_{2}$ escape away to infinity from the singularity with a definite tangent. The non-spacelike curve $\gamma_{3}$, which is emitted after the event horizon, crosses the apparent horizon and falls back to the singularity. Now,

$$
\begin{aligned}
& 2+\lambda X_{0}^{2}-X_{0}=0 \\
& X_{0}=a_{ \pm}=\frac{1 \pm \sqrt{1-8 \lambda}}{2 \lambda}, \\
& Q\left(X_{0}\right)=0 \Rightarrow 1+L^{2} X_{0}\left(2+\lambda X_{0}^{2}-X_{0}\right)=0 .
\end{aligned}
$$

The values $a_{ \pm}$are only possible if $\lambda \leq \frac{1}{8}$ (figure 4 ). In this case the singularity is naked in the sense that families of future directed non-spacelike geodesics going to infinity terminate at the singularity in the past (Penrose diagram 5).

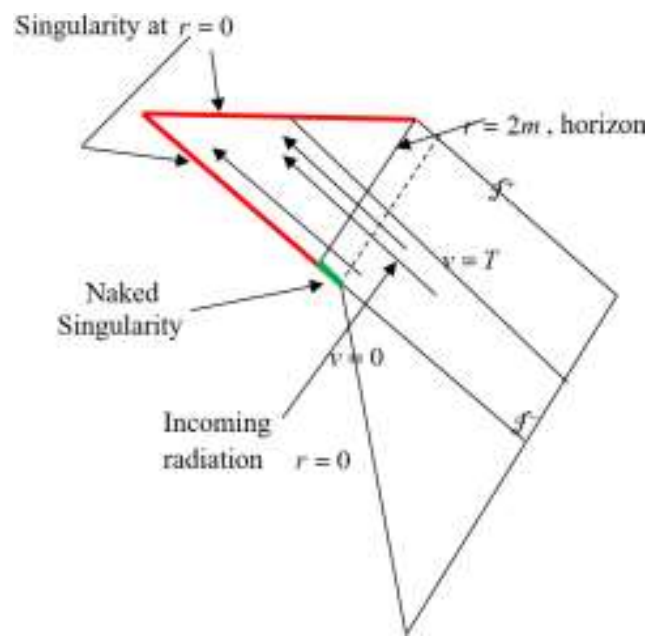

Figure 5: A Penrose diagram for the naked singularity forming in the radiation collapse. 
Now we consider positive sign solutions in equation (30), then;

$$
\frac{d r}{d x}=\frac{r\left[(1+A k)-\left(1+\lambda X^{2}-X\right) Q(X)\right]}{\left(1+\lambda X^{2}-X\right) X Q}
$$

Integrating (35) we get the equation of geodesic curves in $(v, r)$ planes to give $r=r(X)$ for $\lambda \leq \frac{1}{8}$ :

$D r=\frac{1}{X-4} \exp \left(\frac{-4}{X-4}+\int \frac{-L^{2}+\left(\frac{A}{C}\right) r^{2}}{\mathrm{Q}(\mathrm{Q}+1+\mathrm{Ak})} d X\right)$ for $\lambda=\frac{1}{8}$

$D r=\frac{\left(X-a_{+}\right)^{a_{-} /\left(a_{+}-a_{-}\right)}}{\left(X-a_{-}\right)^{a_{+} /\left(a_{+}-a_{-}\right)}} \exp \left(\int \frac{-L^{2}+\left(\frac{A}{C}\right) r^{2}}{\mathrm{Q}(\mathrm{Q}+1+\mathrm{Ak})} d X\right)$ for $\lambda<\frac{1}{8}$

where $D$ is a constant which labels different geodesics in the $(v, r)$ plane. For example, $D=0$ or $D=\infty$ implies $X=\frac{v}{r}=$ constant and gives rise to geodesic which are rectilinear (straight lines) in the $(v, r)$ plane.

For families of non-rectilinear geodesics i.e., for $D \neq 0, \infty$, terminating at $r=0$, the allowed values of $X$ one obtained by simply letting $r \rightarrow 0$ in equation (37) and finding the values of $X$. It follows that either $X=a_{+}$, or $X=c$, where $c$ is a double root of the equation (24). From equation (37) gives the equation of geodesic curves, that $r=0$ implies either;

$$
X=a_{+} \text {or } \exp \left(\int-\frac{d X}{Q(X)}\right) \rightarrow 0,
$$

which means $Q(X) \rightarrow 0$ for some real values of $X=X_{c}$. That means $X_{c}$ is a root of equation (34), a third degree equation, so has three roots; however since $L^{2}, \lambda>0$, hence it has only two positive and one negative roots. If all the roots are distinct then,

$$
\int \frac{d X}{Q(X)} \cong \int \frac{d X}{\sqrt{X-X_{c}}} \rightarrow \infty
$$

and so, $r=0$ at $X=X_{c}$. The double root exists if and only if; 


$$
L^{2} C^{2} \equiv \ell^{2}=\ell_{c r t}^{2}=\frac{27 C^{2} \lambda^{2}}{2\left(1-9 \lambda+(1-6 \lambda)^{1 / 2}\right)}
$$

For the radial case when $\ell=0$,

$$
\begin{aligned}
& 2+\lambda X^{2}-X=0 \\
& X=\frac{1 \pm \sqrt{1-8 \lambda}}{2 \lambda}=a_{ \pm}, \\
& K^{v}=2 C^{\prime} k^{\left(2-a_{ \pm}\right) / a_{ \pm}}, K^{r}=\frac{2 C^{\prime}}{a_{ \pm}} k^{\left(2-a_{ \pm}\right) / a_{ \pm}}, \\
& r=C^{\prime} k^{2 / a_{ \pm}}, v=C^{\prime} a_{ \pm} k^{2 / a_{ \pm},}
\end{aligned}
$$

where $C^{\prime}$ is a constant. For the non-radial rectilinear geodesic we get;

$$
\begin{aligned}
& 2+3 \lambda X^{2}-2 X=0 \\
& X=\frac{1+\sqrt{1-6 \lambda}}{3 \lambda}=c, \\
& K^{v}=\frac{\ell}{r \sqrt{2 \lambda c-1}}, \\
& K^{r}=\frac{\ell}{c r \sqrt{2 \lambda c-1}}, \\
& r^{2}=\frac{2 \ell k}{c \sqrt{2 \lambda c-1}}, \text { and } \\
& X=\frac{v}{r}=c .
\end{aligned}
$$

The rectilinear geodesics meet the initial point of the singularity $v=0, r=0$ provided $a_{ \pm}$, and the tangents are real, which implies $\lambda \leq \frac{1}{8}$. The three null lines $X=a_{+}$, $X=a_{-}$, and $X=c$ in the $(v, r)$ plane represent the three rectilinear singular geodesics that terminated at the singularity with either $X=a_{+}$or $X=c$. Here $a_{-}$is a single radial geodesic in the $(v, r)$ plane meeting the singularity, which is not a tangent to any families of non-spacelike geodesics terminating at the naked singularity. So, we have three types of singularities at $v=0, r=0$ as;

$$
X=a_{+}, X=a_{-} \text {, and } X=c
$$


where for $\lambda=\frac{1}{8}, a_{+}=a_{-}=c$. Now we describe the behavior of singular geodesics in terms of the four different regions of the space-time. In region $I$ characterized by $X \geq \frac{1}{\lambda}$ all geodesics are ingoing and no geodesics escape. Here $X=\frac{1}{\lambda}$ is the apparent horizon. Within region $I I, \frac{1}{\lambda}>X>a_{+}$, the region $\frac{(1+\sqrt{1-4 \lambda})}{2 \lambda}>X>a_{+}$is free for all region where all geodesics with values of $\ell$ escape from the singularity with $X=a_{+}$. However for $X>\frac{(1+\sqrt{1-4 \lambda})}{2 \lambda}$ family of geodesics with $\ell^{2}<\frac{(1-\lambda X)}{\left(1+\lambda X^{2}-X\right)^{2}}$ escape while others fall back to $r=0$.

Region III, $a_{+}>X>a_{-}$, is separated from region $I I$ by the rectilinear radial null geodesic $X=a_{+}$. All families of geodesics with $\ell<\ell_{c r t}$ all over this region have their past end point at singularity with $a_{+}>X>c$, while geodesics with $\ell=\ell_{c r t}$ within this region have their past end point at singularity with tangent $X=c$, which is the rectilinear nonradial geodesic. In region $I V$, no non-spacelike geodesic has an end point at the singularity in the past. Now we consider the following scalar for the Vaidya space-time,

$\psi=R_{\mu v} K^{\mu} K^{v}=\frac{\lambda}{r^{2}}\left(K^{v}\right)^{2}$

where $R^{\mu v}$ is the Ricci curvature tensor. For non-spacelike geodesics the geodesic equations are given by;

$$
\begin{aligned}
& \psi=\frac{\lambda C^{2}(1+B C k+Q)^{2}}{r^{4}\left(2+\lambda X^{2}-X\right)^{2}} \\
& =\frac{4 \lambda C^{2}(1+B C k)^{2}}{r^{4}\left(2+\lambda X^{2}-X\right)^{2}}+\frac{\left(L^{2}-B r^{2}\right) \lambda X C^{2}(Q(X)+3+3 B C k)}{r^{4}\left(2+\lambda X^{2}-X\right)^{2}(Q(X)+1+B C k)} .
\end{aligned}
$$

By (37), equation (46) gives;

$$
\operatorname{Lim}_{k \rightarrow 0} k^{2} R_{\mu \nu} K^{\mu} K^{v}=\operatorname{Lim}_{k \rightarrow 0} k^{2} \psi=\lambda C^{2}\left[\operatorname{Lim}_{k \rightarrow 0}(1+B C k+Q(X))^{2}\left(\operatorname{Lim}_{k \rightarrow 0} \frac{k^{2}}{r^{4}\left(2+\lambda X^{2}-X\right)^{2}}\right)\right] .
$$

Using L' Hospital rule in equation (36) we get;

$$
\operatorname{Lim}_{k \rightarrow 0} k^{2} \psi=\lambda\left(\frac{\left(1+\sqrt{1+L^{2} X_{0}\left(2+\lambda X_{0}^{2}-X_{0}\right)}\right) X_{0}}{2+\left(X_{0}-2\right) \sqrt{1+L^{2} X_{0}\left(2+\lambda X_{0}^{2}-X_{0}\right)}}\right)^{2}
$$

where $\infty \geq X_{0} \geq-\infty, \operatorname{Lim}_{k \rightarrow 0} k^{2} \psi>0$, hence the strong curvature condition is satisfied along all singular geodesics. 
From equation (26) and (27) we get;

$$
\frac{d X}{d k}=\frac{1}{r}\left(\frac{d v}{d k}-X \frac{d r}{d k}\right)=\frac{Q(X)}{r^{2}} \Rightarrow k=\int \frac{r^{2}(X) d X}{Q(X)}
$$

The non-rectilinear geodesics meet the singularity with either $X=a_{+}$or $r=0, X=c$. For families of geodesics with $X=a_{+}$at the singularity,

$$
\begin{aligned}
& X-a_{+} \boldsymbol{\alpha} k^{n}, n=\sqrt{1-8 \lambda}, r \boldsymbol{\alpha} k^{(1-n) / 2}, \\
& \psi \approx \frac{4 \lambda}{k^{2}}+\frac{2 \ell^{2} D}{k^{(2-n)}},
\end{aligned}
$$

where $D$ is a constant which is negative in region $I I$ and positive in region $I$.

For the class $X=a_{+}$, for radial singular geodesics the behavior of $\psi$ is given by;

$$
\psi \approx \frac{4 \lambda}{k^{2}}, \text { and } \operatorname{Lim}_{k \rightarrow 0} k^{2} \psi=4 \lambda
$$

For all other geodesics $(\ell \neq 0)$, we have again;

$\psi \approx \frac{4 \lambda}{k^{2}}+\frac{2 \ell^{2} D}{k^{(2-n)}}$

and again $\operatorname{Lim}_{k \rightarrow 0} k^{2} \psi=4 \lambda$.

For $X=c$ the behavior of curvature scalar $\psi$ for the rectilinear non radial trajectories are given by $\psi \approx \frac{\lambda c^{2}}{4 k^{2}}$, and $\operatorname{Lim}_{k \rightarrow 0} k^{2} \psi=\frac{\lambda c^{2}}{4}$. For non-radial trajectories in this class, is given by $\ell=\ell_{c r t}$, we have $\psi \approx \frac{\lambda c^{2}}{4 k^{2}}$, which gives $\operatorname{Lim}_{k \rightarrow 0} k^{2} \psi=\frac{\lambda c^{2}}{4}$.

Finally, for the rectilinear radial geodesic with $X=a_{-}$, we have $\psi \approx \frac{4 \lambda}{k^{2}}$ and $\operatorname{Lim}_{k \rightarrow 0} k^{2} \psi=4 \lambda$, which satisfy strong curvature condition. Now we examine the behavior of the Kretschmann scalar near the singularity forming in the Vaidya-Papapetrou models. The Kretschmann scalar is given by;

$$
\alpha=R^{\mu \nu \lambda \sigma} R_{\mu \nu \lambda \sigma}=\frac{48 m(v)^{2}}{r^{6}}=\frac{12 \lambda^{2} X^{2}}{r^{6}},
$$

along the families of the non-spacelike geodesics joining the singularity. Near the naked singularity for $X=a_{+}$; 


$$
\alpha=\frac{12 \lambda^{2} a_{+}^{2}}{c_{1}^{4} k^{8 / a_{+}}} .
$$

Then, $\operatorname{Lim}_{k \rightarrow 0} k^{2} \alpha=0$ and $\left.\operatorname{Lim}_{k \rightarrow 0} \alpha\right|_{k=k_{0}}=\frac{12}{c_{1}^{4}}$, which turns out that the divergence of $\alpha$ is $\lambda$ dependent. For $X=c$ we get;

$\alpha=\frac{12 \lambda^{2} c^{4}(2 \lambda c-1)}{\ell_{c r t}^{2} k^{2}}$

and $\operatorname{Lim}_{k \rightarrow 0} k^{2} \alpha=\frac{12 \lambda^{2} c^{4}(2 \lambda c-1)}{\ell_{c t}^{2} k^{2}} \quad$ where $\left.\operatorname{Lim}_{k \rightarrow 0} \alpha\right|_{k=k_{0}}=0$ and the divergence of $\alpha$ turns out to be $\lambda$ independent.

Finally for $X=a_{-}, \alpha=\frac{12 \lambda^{2} a_{-}^{2}}{c_{1}^{4} k^{8 / a_{-}}}$, again implies divergence of $\alpha$ be $\lambda$ dependent and bounded by $\lambda \leq \frac{1}{8}$.

It is clear that naked singularity exhibits a directional behavior as far as the Kretschmann scalar is concerned along different families.

By the above discussion we can remark that not just isolated trajectories but entire families of non-spacelike geodesics escape from the naked singularity at the origin outside the event horizon and inside the Cauchy horizon $X=a_{-}$. As a result, the naked singularity is visible to a far away observer for an infinite period of time once he or she has received the first ray from the singularity, and hence it is a strong curvature naked singularity in a very powerful sense.

\section{Concluding Remarks}

In this study we have briefly described general relativity, definitions of singularities and the gravitational collapse of a massive star. When a black hole is created due to an endless gravitational collapse of a massive star in the space-time, it covers the space-time singularity, which is called the cosmic censorship hypothesis. We have discussed the naked singularities by the Vaidya space-time metric with sufficient mathematical calculations. Throughout the paper we have avoided difficult mathematical calculations and have displayed diagrams where necessary.

\section{REFERENCES}

Christodoulou, D. (1999), Ann. Math. 149, 183.

Clarke, C.J.S. (1993), An Analysis of Space-time Singularities, Cambridge University Press, Cambridge.

Deshingkar, S.S. (2010), On Physical Insignificance of Null Naked Singularities, arXiv:1012.3090v1 [gr-qc] 14 Dec 2010.

Dwevedi, I.H. and Joshi, P.S. (1989), On the Nature of Naked Singularities in Vaidya Space-time, Class. Quantum Grav., 6:1599. 
Dwevedi, I.H. and Joshi, P.S. (1991), On the Nature of Naked Singularities in Vaidya Space-time II, Class. Quantum Grav., 8:1339.

Harada, T. (2004), Pramana, Proc. ICGC-2004 63, 741.

Harada, T.; Iguchi, H.; and Nakao, K.-I. (2002), Physical Processes in Naked Singularity Formation, Progress of Theoretical Physics, 107(3): 449-524.

Hawking, S.W. and Ellis, G.F.R. (1973), The Large Scale Structure of Space-time, Cambridge University Press, Cambridge.

Hiscock, W.A.; Williums, L.G. and Eardeley, D.M. (1982), Creation of Particles by Self- focusing Singularities, Phys, Rev. D., 26: 751.

Joshi, P.S. (1996), Global Aspects in Gravitation and Cosmology, $2^{\text {nd }}$ Ed., Clarendon Press, Oxford.

Joshi, P.S. (2013), Spacetime Singularities, arXiv:1311.0449v1 [gr-qc] 3 Nov 2013.

Kr'olak, A. (1999), Nature of Singularities in Gravitational Collapse, Progress of Theoretical Physics Supplement No. 136.

Kruskal, M.D. (1960), Maximal Extension of Schwarzschild Metric, Physical Review, 119(5): 1743-1745.

Kurada, Y. (1984), Naked Singularities in Vaidya Space-times, Prog. Theor. Phys., 72: 63.

Mkenyeleye, M.D.; Goswami, R. and Maharaj, S.D. (2014), Gravitational Collapse of Generalised Vaidya Spacetime, arXiv:1407.4309v2 [gr-qc] 29 Sep 2014.

Mohajan, H. (2015). Basic Concepts of Differential Geometry and Fibre Bundles. ABC Journal of Advanced Research, 4(1), 57-74. Retrieved from http://iproclaim.my/archive/index.php/abcjar/article/view/200

Mohajan, H. (2015). Generalization of Euler and Ramanujan's Partition Function. Asian Journal Of Applied Science And Engineering, 4(3), 167-190. Retrieved from http://journals.abc.us.org/index.php/ajase/article/view/681

Mohajan, H. (2016). Singularities in Global Hyperbolic Space-time Manifold. Asian Journal Of Applied Science And Engineering, 5(1), 41-58. Retrieved from http://journals.abc.us.org/index.php/ajase/article/view/815

Mohajan, H. (2017). Tacit Knowledge for the Development of Organizations. ABC Journal of Advanced Research, 6(1), 17-24. Retrieved from http://iproclaim.my/archive/index.php/abcjar/article/view/263

Mohajan, H.K. (2013a), Singularity Theorems in General Relativity, M. Phil. Dissertation, Lambert Academic Publishing, Germany.

Mohajan, H.K. (2013b), Friedmann, Robertson-Walker (FRW) Models in Cosmology, Journal of Environmental Treatment Techniques, 1(3): 158-164.

Mohajan, H.K. (2013c). Schwarzschild Geometry from Exact Solution of Einstein Equation, Journal of Environmental Treatment Techniques, 1(2): 69-75.

Mohajan, H.K. (2014a), Upper Limit of the Age of the Universe with Cosmological Constant, International Journal of Reciprocal Symmetry \& Theoretical Physics, 1(1): 43-68.

Mohajan, H.K. (2014b), Gravitational Collapse of a Massive Star and Black Hole Formation, International Journal of Reciprocal Symmetry \& Theoretical Physics, 1(2): 125-140.

Papapetrou, A. (1985), Formation of a Singularity and Causality in a Random Walk in Relativity and Cosmology (Eds. N. Dadhich; J. K. Rao; J. V. Narlikar and C. V. Vishveshwara) Wiley Eastern, New Delhi.

Penrose, R. (1969), Riv. Nuovo Cim. 1, 252.

Singh, T.P. (1997), Singularities and Cosmic Censorship, J. Astrophys. Astr., 18: 335-338.

Szekeres, G. (1960), On the Singularities of a Riemannian Manifold, Publicationes Mathematicae, Debrecen. 7: 285-301. 
Vaidya, P.C. (1943), The External Field of a Radiating Star in General Relativity, Curr. Sci. 12: 183.

Vaidya, P.C. (1951), The Gravitational Field of a Rotating Star, Proc. Of the Indian Acad. Sci. A33: 264.

Witten, E. (1992), hep-th/9206069. 